\title{
ASIMETRÍAS EN LOS SISTEMAS EDUCATIVOS Y EN LOS PATRONES INSTITUCIONALES: HACIA LA CONSTRUCCIÓN DE UN INDICADOR GLOBAL DE CAPITAL HUMANO*
}

\author{
JORGE E. SÁENZ C.**; GERMAN SÁNCHEZ P.**** \& LUZ AYDÉE HIGUERA C.****** \\ UNIVERSIDAD DE TORONTO (CANADÁ) - UNIVERSIDAD MILITAR NUEVA GRANADA - \\ UNIVERSIDAD LA GRAN COLOMBIA
}

Recibido/ Received/ Recebido: 21/09/2016 - Aceptado/ Accepted / Aprovado: 10/11/2016

\begin{abstract}
Resumen
En este trabajo se presenta una revisión teórica y empírica sobre la relación capital humano y crecimiento económico. Se encuentra que existe una relación del capital humano con otros factores, como la calidad de la educación, la estabilidad política, la confiabilidad del sistema judicial, la corrupción y la burocracia. Igualmente, se presenta un análisis acerca de las consideraciones teóricas del capital humano, resaltando las divergencias estadísticas presentadas en los coeficientes estimados, cuando se toman distintas variables para capturar los efectos de este factor sobre el producto. Además, se hace una revisión teórica sobre la importancia del capital humano en la productividad, y se analiza las asimetrías institucionales con las estadísticas vigentes para 37 países. Por último, se propone y se calcula un Indicador Global de Capital Humano (IGCH).

Palabras clave: Capital humano; Crecimiento económico; Escolaridad; Calidad de la educación; Indicador Global de Capital Humano.
\end{abstract}

\section{ASYMMETRIES IN EDUCATIONAL SYSTEMS AND INSTI- TUTIONAL PATTERNS: TOWARDS THE CONSTRUCTION OF A HUMAN CAPITAL GLOBAL INDICATOR}

\begin{abstract}
This paper presents a theoretical and empirical review of the relationship between human capital and economic growth. It is found that there is a relationship between human capital and other factors, such as the quality of education, political stability, the reliability of the judicial system, corruption and bureaucracy. Likewise, an analysis is presented about the theoretical considerations of human capital, highlighting the statistical divergences presented in the estimated coefficients,

\footnotetext{
Este trabajo se deriva de las diferentes investigaciones que han realizado los autores en las universidades de Toronto -Canadá-, Militar Nueva Granada y Uniciencia -Colombia-, y sus inquietudes sobre un tema tan fundamental como el crecimiento económico y el capital humano. Se agradecen los comentarios de los profesores Albert Berry, Jorge Iván Gonzales y Edgar Revéis

* Economista y magister en Economía. Consultor e investigador independiente. Actualmente es Asistente del Profesor Albert Berry de la Universidad de Toronto. Correo electrónico: josaenza8@gmail.com

*.*. Doctor en Ciencias Económicas. Profesor e investigador de la Universidad Militar Nueva Granada Correo electrónico: german.sanchez@unimilitar.edu.co

Economista y Estudiante de Maestría en Relaciones y Negocios Internacionales, Universidad Militar "Nueva Granada". Correo electrónico: aydeehiguera@gmail.com
} 
when different variables are taken to capture the effects of this factor on the product. In addition, a theoretical review is made on the importance of human capital in productivity, and institutional asymmetries are analyzed with current statistics for 37 countries. Finally, a Human Capital Global Indicator (IGCH) is proposed and calculated.

Keywords: Human capital; Economic growth; Education; Quality of education; Human Capital Global Indicator.

\title{
ASSIMETRIAS NOS SISTEMAS EDUCATIVOS E NOS PA- DRÕES INSTITUCIONAIS: PARA A CONSTRUÇÃO DE UN INDICADOR GLOBAL DE CAPITAL HUMANO
}

\begin{abstract}
Resumo
Neste trabalho apresenta-se uma revisão teórica e empírica sobre a relação capital humano e crescimento econômico. Encontra-se que existe uma relação do capital humano com outros fatores, como a qualidade da educação, a estabilidade política, a fiabilidade do sistema judicial, a corrupção e a burocracia. Igualmente, apresenta-se uma análise a respeito das considerações teóricas do capital humano, realçando as divergências estatísticas apresentadas nos coeficientes estimados, quando se tomam diferentes variáveis para capturar os efeitos deste fator sobre o produto. Além do mais, faz-se uma revisão teórica sobre a importância do capital humano na produtividade, $e$ analisam-se as assimetrias institucionais com as estatísticas vigentes para 37 países. Por último, propõe-se e calcula-se um Indicador Global de Capital Humano (IGCH).

Palavras chave: Capital humano; Crescimento econômico; Escolaridade; Qualidade da educação; indiCador global de capital humano.
\end{abstract}

Sáenz, J., Sánchez, G. \& Higuera, L. (2018) Asimetrías en los sistemas educativos y en los patrones institucionales: hacia la Construcción de un Indicador Global de Capital Humano. En: Revista de la Facultad de Ciencias Económica: Investigación y Reflexión. rev.fac.cienc. econ, XXVI (1), DOI: https://doi.org/10.18359/rfce.3146

JEL: I25, 015 .

\section{Introducción}

Una particularidad que ostentan las teorías de crecimiento económico tradicional desarrolladas en las últimas décadas ha sido el enriquecimiento del concepto capital humano. En los modelos neoclásicos tradicionales, por ejemplo, se concentraban primordialmente en la acumulación de capital físico -maquinaria e infraestructura-, mientras en los modelos que se han desarrollado en los últimos años le dan mayor énfasis a la acumulación de capital humano ${ }^{1}$. Es de resaltar, sin embargo, que la evidencia

1 Un análisis del tema se puede encontrar en los estudios de Barro (1991), y Caballé \& Santos (1993). empírica que se ha obtenido con series estadísticas para diferentes países, no ha sido consistente con los modelos teóricos desarrollados. En efecto, el resultado de los ejercicios econométricos incluyendo la variable -la proxy- capital humano, generalmente presenta coeficientes con signos contrarios a los esperados y con poca robustez estadística; especialmente, cuando se estiman regresiones empleando especificaciones en diferencias o con datos panel ${ }^{2}$.

\footnotetext{
Es un modelo de regresión que utiliza, para la estimación de los parámetros de interés, la variabilidad temporal y transversal de los datos. Para obtener un análisis más completo de este tema se puede revisar el artículo de Dessus (2001) "Human capital and growth the recovered role of educational systems".
} 
La persistencia de estos resultados en diferentes investigaciones ha alimentado un creciente escepticismo en este tema ${ }^{3}$, lo que ha llevado a exponer diversas razones para tratar de explicar ¿el por qué las variables que tratan de capturar los efectos de la inversión en capital humano sobre el crecimiento del PIB, arrojan resultados distintos a los esperados? (Pritchett, 1996). Una hipótesis que ha cobrado preeminencia recientemente, es que los malos resultados estadísticos que se obtienen en las estimaciones econométricas son producto de la mala calidad de los datos de escolarización que se han empleado como variable proxy del capital humano (Johannes, 1998).

Para analizar el problema con mayor énfasis, tendríamos que partir de la ecuación de producción ${ }^{4}$. En efecto, al tomar el lado derecho de dicha ecuación, manteniendo las demás variables constantes -céteris páribus-, se puede advertir que las variaciones que afectan al PIB, dependen de los cambios que produzca el capital humano, de modo que si no se hace una buena selección de dicha variable $y$ se incorporan los factores que la afectan -como, por ejemplo, los rezagos en la calidad de la educación que están presentes en los sistemas educativos de los países-, las estimaciones econométricas que se obtengan no serán robustas y los parámetros y elasticidades que se calculen tendrán errores de estimación.

El problema se podría plantear igualmente si lo evaluamos desde el lado izquierdo de la ecuación: el PIB presenta una gama de problemas en su medición, no solamente porque deja por fuera muchos sectores que contribuyen a la economía ${ }^{5}$, sino porque, además, no tiene en cuenta los ingresos provenientes por actividades ilegales -narcotráfico, corrupción, delincuencia común, etc.-, que si bien podrían contribuir a empujar muchos renglones de la economía, de igual modo generan un daño social

\footnotetext{
$3 \quad$ Véase por ejemplo Pritchett (1996)

4 La función de producción está definida por la siguiente ecuación: $Y=A K^{\alpha} L^{\beta} H^{\delta}$, donde $\mathrm{Y}=\mathrm{PIB}, \mathrm{A}=$ Tecnología, $\mathrm{K}=$ Capital Fisico, $\mathrm{L}=$ Fuerza laboral y $\mathrm{H}=$ Capital Humano, $\alpha, \beta$ y $\delta$ son las elasticidades.

5 Por ejemplo, no se tiene en cuenta la economía del cuidado
}

y afectan la ecuación ${ }^{6}$. El mismo problema lo produce la no incorporación de los efectos que genera el agotamiento de los recursos naturales y el deterioro de la calidad ambiental ${ }^{7}$.

En este marco, este documento tiene dos objetivos principales: primero, identificar los sesgos de estimación que presenta la ecuación de producción cuando no se tiene en cuenta las asimetrías de la calidad de la educación y los factores institucionales que afectan el valor productivo del capital humano; $y$, segundo, construir un indicador global de capital humano, que permita contar con una medida más integral y acorde con los precedentes que acompañan a la teoría del capital humano.

El documento contiene 5 secciones, incluyendo esta introducción. En la segunda sección se presenta una revisión teórica y empírica sobre la importancia del capital humano y su relación con otros factores como la calidad de la educación, la estabilidad política, la confiabilidad del sistema judicial, la corrupción, la facilidad para emprender nuevas iniciativas empresariales y la burocracia ${ }^{8}$, todo conducente al cálculo de un Indicador Global de Capital Humano (IGCH).

En la tercera sección se hace un análisis acerca de las consideraciones teóricas del capital humano, resaltando la divergencia estadística que se presenta en los coeficientes estimados cuando se toman distintas variables para capturar la contribución del ca-

6 En el caso de Colombia, los últimos hallazgos de Reveiz (2014) reflejan una participación de la sociedad ilegal criminal en el PIB del $8 \%$.

7 De acuerdo con los últimos estudios Banco Mundial anualmente se gasta por concepto de capital natural alrededor del $12 \%$ del PIB. Esto nos da una idea de las presiones que nuestro sistema productivo ejerce sobre el medio ambiente y la importancia de medirlas de alguna manera. Si nosotros pudiéramos socializar esto, si pudiéramos comunicar cuál es el daño ambiental que se está llevando a cabo al producir y al consumir, seguramente podríamos negociar con las instancias competentes para lograr una mejor asignación de recursos financieros, materiales y humanos.

8 En este estudio no incluimos variables de tipo social, ni cognitivas que son consideradas como dotaciones iniciales de los individuos. 
pital humano en el producto 9 . Igualmente, se hace una revisión teórica de la importancia del capital humano en la productividad. También se compilan los datos de escolaridad y puntaje obtenido en las pruebas de matemáticas y ciencias para 42 países $^{10}$. Finalmente, se analiza las asimetrías institucionales con las estadísticas vigentes para 37 países. La cuarta sección, describe teórica y matemáticamente, la metodología y los resultados del cálculo del Indicador Global de Capital Humano. Y, en la quinta sección, se ofrecen las conclusiones finales.

\section{Conceptualización, definición y pro- blemas}

\subsection{Preeminencia y evolución del capital huma- no en el crecimiento económico}

El capital humano es uno de los factores que afecta directamente a la producción, debido a que determina la calidad del trabajo, lo que se traduce en aumentos o pérdidas en la productividad. Fue a partir de esta aseveración que los economistas empezaron a considerar todo lo relacionado con la acumulación de capital humano como una rama importante de la teoría del crecimiento, comenzando con los trabajos pioneros de Becker (1962, 1964), Schultz (1960, 1961, 1962), Denison (1962, 1964), Nelson \& Phelps (1966), Blaug (1976). Estos autores ofrecieron las primeras aproximaciones sobre la importancia del capital humano como un factor importante a incluir en la función de la producción, aunque, de cierto modo, se ha reconocido que Adam Smith, en su libro sobre la Riqueza de las naciones (1776), fue el primero en señalar que la acumulación de capital es la clave para lograr mayores tasas de crecimiento económico ${ }^{11}$. No obstante, no fue sino hasta la aparición de los modelos de crecimiento endógeno, desarrollados por Lucas (1988, 1993), Romer (1990), King \& Rebelo (1991), Stokey (1991),

$9 \quad$ En este caso se utilizan variables proxy. Una variable proxy es la que está relacionada con una variable no observable que se desee incluir en un modelo.

10 Esta información se obtuvo de la Prueba PISA (2013)

11 Los orígenes del Capital Humano también se pueden retrotraer de otros autores como: Senior (1986), StuartMill(1864) y John Baptiste Say (1880).
Young (1991), y Acemoglu (1997), que se le da importancia mayúscula al capital humano.

Romer (1990) afirma que "una economía con un stock mayor de capital humano va a experimentar un crecimiento más rápido", y lo propone como el elemento clave del sector de la investigación que genera nuevas ideas y los productos que forman la base técnica de los avances. Este mismo autor llega a sugerir que el libre comercio internacional acelerará el crecimiento y que bajos niveles de capital humano pueden ser explicados por menores tasas de crecimiento en los países menos desarrollados, que a menudo son más cerrados al comercio internacional. Grossman \& Helpman (1991) siguen la misma línea de razonamiento, haciendo hincapié en que una mayor concentración de la cuenta de capital humano para el crecimiento de la actividad de investigación, explica el aumento de la cantidad de innovación que se origina en un país determinado.

Chari \& Hopenhayn (1991) desarrollaron un modelo teórico en la línea de los modelos "clásicos", señalando que cada tecnología requiere de un elemento humano especialmente cualificado y que la capacidad global de la mano de obra tiene una influencia importante en la velocidad con que las nuevas tecnologías son adoptadas y usadas.

Barro (1991), en un estudio de corte transversal, utilizando una muestra para 98 países durante el período 1960-1985, concluye que la tasa de crecimiento del PIB per cápita está positivamente relacionada con la cantidad de recursos humanos del capital presente inicialmente. Como variables proxy de capital humano, utiliza los índices de escolarización en la enseñanza primaria y secundaria para $1960^{12}$

Mankiw, Romer \& Weil (1992), con datos panel y una muestra de 98 países para el periodo 19601985, emplean como variable proxy del capital

12 Ante la imposibilidad de utilizar una variable de stock debido a la falta de disponibilidad de datos comparables en todos los países de la muestra, se usa una variable del flujo, aunque con retraso en el examen de los índices de éstos pueden mostrar un efecto similar a la de una variable stock. 
humano, el porcentaje de población en edad de trabajo que han completado la educación secundaria y encuentran que, a pesar del hecho de que esta variable tiene algún efecto en los ingresos per cápita, es menos del 30 por ciento.

Eicher (1996) se refirió a la necesidad de mano de obra calificada para adaptar las nuevas tecnologías al proceso productivo en los sectores de alta tecnología; sin embargo, afirmó que una vez que esta ha sido absorbida, existe la necesidad de utilizar menos mano de obra calificada.

La importancia del capital humano también fue desarrollada ampliamente por otros economistas notables como: Azariadis \& Drazen(1990), Levine \& Renelt(1992), Kyriacou (1991, 1992), Barro \& Lee (1997), Benhabid \& Spiegel (1994, 1997), Mingat \& Ten (1996), Krueger \& Lindahl (1999), Tamura (1991), Freesman Polasky (1992), Caballé \& Santos (1993), Caballé (1995), entre otros. La mayoría de estos autores coinciden en que la inversión en capital humano no sólo aumenta la productividad, sino que también conduce a mayores niveles de vida.

Sachs (1997), Blanchard (1997), Mankiw (2009), Dornbusch, Fisher \& Startz (1998) en sus famosos libros de macroeconomía que han circundando el mundo por muchos años, expresan que los países que no aumentan sus inversiones en educación y salud, tendrán dificultades para el logro de mayores tasas de crecimiento económico y, por lo tanto, no son susceptibles de mejorar de manera significativa.

\subsection{Definiciones y mediciones de capital humano}

Para tener una idea del significado que tiene para los economistas el capital humano, es necesario hacer una recopilación de las más importantes definiciones que han hecho algunos estudiosos del tema sobre este concepto.

Como acabamos de resaltar, las consideraciones iniciales sobre capital humano provienen de Smith (1776), Senior (1986), Stuart-Mill (1864), John Baptiste Say (1880), Cannan (1928) y Wal- sh (1935), quienes resaltan que las habilidades o conocimientos de los trabajadores y la formación influyen en la productividad del trabajo $y$, consecuentemente, en los ingresos. No obstante, las consideraciones teóricas formales del capital humano se encuentran en los trabajos pioneros de Becker y Mincer. Becker en sus estudios de (1962, 1964) definió el capital humano como el conjunto de las capacidades productivas que un individuo adquiere por acumulación de conocimientos generales o específicos que puede ser acumulado, o usarse. Mincer, por su parte, resaltó el efecto que ejerce la capacitación o aprendizaje en el trabajo en el aumento de los ingresos.

Trabajos relativamente recientes de economistas como Lucas (1988) y Uzawa (1965) definen el capital humano como el conocimiento acumulado que consiguen los individuos cuando adquieren educación. El concepto de conocimiento acumulado puede implicar consideraciones más relacionadas con la calidad (o resultado) de la experiencia educativa. En dicho estudio los autores muestran cómo, en once estudios empíricos, se utilizaron diferentes variables para medir el capital humano. Las variables utilizadas fueron: matricula, los años de escolaridad, alfabetización, entre otras ${ }^{13}$.

Romer (1990), define "el capital humano como aquel que incluye la suma de las capacidades que tienen influencia en la producción y que se incorporan en las personas o colectivos: la educación (habilidades y conocimientos, actitudes generales), la formación profesional (conocimientos y capacidades técnicas), la salud, las virtudes comunes de vida, etc.”. Barro \& Sala i Martin (1995), muestran

3 La corriente preocupación por la mala calidad de los datos educativos y por las distorsiones que estos generan en el momento de estimar los efectos del capital humano sobre el producto, ha motivado a muchos economistas a intentar mejorar el contenido informativo de las series de escolarización a través de la explotación de nuevas fuentes y la introducción de diversas correcciones. La existencia de errores de medición genera una tendencia a la infravaloración del impacto del capital humano sobre la productividad. Intuitivamente, el sesgo surge debido generalmente a que los errores de medición suelen traer "ruido" que tiende a oscurecer la relación entre las variables de interés. 
que una de las formas más frecuentes de definir y cuantificar el capital humano es mediante el cálculo de los años promedio de escolaridad de la población activa.

Si tenemos en cuenta la ambigüedad actual en torno al capital humano y su definición, no sorprende ver que los economistas aún no lo han considerado en términos de la calidad de la variable. Dornbusch, Fisher \& Startz (1998) resaltaron en sus libros de Macroeconomía que, si bien el capital humano es difícil de medir con precisión, el número de años promedio de estudio puede ser una buena representación aproximada de la definición de capital humano.

En conclusión, existen diferencias en la definición y en la medición del capital humano. La mayoría de los economistas se conforman con afirmar que el capital humano es un elemento cuantificable en el crecimiento económico, y consideran que este varía no sólo en cantidad sino también en calidad.

\subsection{Sesgo en la medición del capital humano}

Como se ha resaltado, uno de los problemas relacionados con la teoría de capital humano que mayor atención ha merecido en los últimos años es que, a pesar de la relevancia que tiene este factor en el crecimiento económico, no hay un consenso sobre ¿qué es realmente el capital humano y cómo medirlo?

Estos interrogantes tienen antecedentes desde los tiempos de Marshall (1961), Shultz (1961) y Chamberlain (1967), quienes se opusieron firmemente a que la inversión en personas se comparara con la inversión no humana. Marshall (1961) rechazó el concepto del capital humano partiendo de dos premisas: la primera se fundamenta en que el trabajador que vende su mano de obra retiene para sí la propiedad de sus facultades, lo que genera que los gastos en formación que hacen los empresarios para sus empleados, nunca serán recuperados; la segunda premisa, sostiene que la educación previa que hayan recibido los trabajadores es producto de la previsión y bondad de los padres; así, los gastos de inversión en personas deben ser considerados como externalidades y no equiparados a una decisión de inversión.

Shultz (1961) manifiesta su rechazo al concepto considerando que si se aplica el concepto de capital al trabajo, intrinsecamente se equipara el hombre con la máquina, lo que lo reduce a un componente meramente material. Chamberlain (1967) sienta su oposición esgrimiendo que el término capital humano equipara implícitamente a los trabajadores con bienes de equipo en los que invertir, lo que conlleva a comparar el rendimiento de la inversión en educación con el rendimiento en inversiones alternativas como bienes físicos tales como carreteras, sistemas de irrigación, etc. Similarmente, el mismo autor aduce que no se pueden calcular tasas de retorno de cualquier inversión a largo plazo debido a la incertidumbre del futuro.

Por su parte, Becker (1998) sostiene que las discusiones que acompañan a la teoría del capital humano, se derivan principalmente porque no existe una medida exacta para cuantificarlo. De hecho, Becker (1998) argumenta que la variable de mayor uso por parte de los economistas en los ejercicios econométricos, años de escolaridad promedio de los trabajadores, no produce automáticamente capital humano. El valor productivo del capital humano depende de la organización, del sistema económico, de las habilidades individuales, y de muchas otras consideraciones. Los años promedios de escolaridad de los trabajadores no es una medida apropiada para hacer comparaciones entre países, debido principalmente a que el costo de producción de ese factor en un año y la productividad del mismo varía de un país a otro. Becker (1998) no piensa que la mayoría de economistas asuman que la educación y el entrenamiento son las medidas más fáciles para intentar capturar el aporte de capital humano en el producto, lo que él esgrime es que comúnmente los economistas la usan como la medida más fácil para conseguir un resultado econométrico. Para Becker (1998), la nutrición, el conocimiento tecnológico y otros recursos de información son más relevantes para la obtención del valor productivo del capital 
humano que la propia formación en el trabajo y la educación formal ${ }^{14}$.

A pesar de los planteamientos de Becker (1998), la mayoría de economistas coinciden en que la variable que más se acerca a la definición de capital humano son los años promedio de escolaridad de los trabajadores. Sin embargo, al chequear los resultados econométricos para una gama de estudios, los coeficientes estimados arrojan diferencias significativas (tabla 1). Por ejemplo, los dos estudios realizados por Barro \& Lee (1997), muestran una diferencia de 0,04. Las mismas diferencias se observan en los resultados de Romer, Mankiw, Islam, Benhabid \& Spiegel, Lau \& Judson ${ }^{15}$.

Tabla 1. Elasticidades de producción de capital humano en Cross-Country regretions

\begin{tabular}{|l|l|l|c|c|}
\hline \multicolumn{1}{|c|}{ Autor } & Model & \multicolumn{1}{|c|}{ h.c. variable } & Coefficient & \multicolumn{1}{c|}{ T-value } \\
\hline Mankiw, Romer \& Weil(1992) & RF & Matriculados & 0.28 & 0.3 \\
\hline Barro \& Lee (1997) & RF & Años Escolaridad & 0.06 & 0.02 \\
\hline Barro \& Lee (1997) & RF & Años Escolaridad & 0.20 & 5.2 \\
\hline \multirow{2}{*}{ Romer (1990) } & RF & Alfabetizacion & $0.24-0.11$ & 2.3 \\
\cline { 2 - 6 } & RF & Años Escolaridad & $-0.01--0.2$ & $0.05-1.8$ \\
\hline Islam (1995): cross-section & RF & Años Escolaridad & $0.09-0.04$ & $2.6-2.0$ \\
\hline Islam (1995): panel estimación & AS & Años Escolaridad & -0.02 & 1.4 \\
\hline WDR 1991 & AS & Enrollmentperp.inv. & $0.02-0.22$ & $0.7-2.8$ \\
\hline Benhabid \& Spiegel(1991) & AS & Varios & 0.02 & 1.6 \\
\hline Benhabid \& Spiegel(1994) & AS & Años Escolaridad & 0.10 & 2.3 \\
\hline Lau, Jamison \& Louat(1991) & AS/RF & Valor & \multicolumn{2}{|c|}{} \\
\hline
\end{tabular}

AS= Augmented Solow, RF=id. Reduced Form. Source: Hers, Johannes(1998)

Fuente: Judson(1993).

Robert Solow en su obra de 1990, argumenta que los salarios y satisfacción en el trabajo están profundamente vinculados a las condiciones sociales prevalecientes y con el concepto, de los trabajadores, de la justicia social. Ningún otro elemento en el campo de las relaciones laborales, tiene un mayor efecto en la reducción de la moral en el lugar de trabajo, en la creación de la insatisfacción personal, en el fomento de ausentismo y la disminución de la productividad, que las desigualdades evidentes en la compensación de los individuos dentro del mismo lugar de trabajo.

14 Estas reflexiones del Profesor Becker hacen parte de una comunicación escrita que se sostuvo con él en diciembre de 1998.
Solow dice "una vez que se admite que el empleo y los salarios están estrechamente vinculados a las condiciones sociales y los factores de autoestima, uno está abandonando el enfoque dado al mercado de trabajo en los libros de texto." (p. 29). Lo que Solow (1990) afirma tajantemente es que existe una gran variedad de condiciones sociales y culturales que tienen una fuerte influencia sobre el desempeño del trabajador $y$, en consecuencia, el capital humano y las condiciones de estos, además de la calidad de la educación, determinan en gran medida su valor productivo.

15 El capital humano está medido con error, de forma que lo que observamos en realidad no es capital humano sino una proxy ruidosa 
El mercado de trabajo, en el sentido global, entonces, no puede interpretarse como cualquier otro mercado, sólo por cuestiones de salario, la oferta y la demanda y los elementos materiales, sino por muchos otros factores que tienen que ver con la organización social, la justicia, juego limpio y la necesidad humana, todo lo cual repercute profundamente en lo que las personas son capaces de hacer y de lo bien que hacen su trabajo.

Por otro lado, Romer (1986) en sus planteamientos sobre ciudades bajo estatuto, aduce que las normas malas restringen el desarrollo. De ahí que en sus múltiples propuestas presenten una gama de elementos técnicos, económicos y normativos vitales para que un país que está con una infinidad de normas malas, salga del estado de pobreza en que está atrapado. Edgar Reveiz ${ }^{16}$ en sus múltiples trabajos sobre triple sociedad muestra que en una sociedad fragmentada y desigual, el ciudadano común no puede acceder al progreso y mucho menos la poca educación que adquiere puede hacer aporte al valor productivo de un país.

En definitiva, existe un sesgo en la definición y medición del capital humano, debido principalmente a que los economistas han obviado en sus modelos econométricos, las diferencias o asimetrías presentes en los sistemas educativos de los países ${ }^{17}$. Otro problema se deriva al no haberse incorporado el ambiente institucional que subyace en cada país y que menoscaba el valor productivo de la educación. No se pueden soslayar las diferencias educativas que existen entre instituciones y países. Si bien es cierto que un economista de la universidad de Pensilvania recibe un papel similar a uno de la Universidad de Chicago que lo acredita como profesional en dicha especialidad, no se puede desconocer las diferencias que existen en calidad entre las dos institucio-

16 Dentro de la diversidad de temas y publicaciones del profesor Edgar Reveiz (2004), el libro sobre El desenlace neoliberal: tragedia o renacimiento, muestra con mayor detalle su pensamiento.

17 Estas diferencias también se dan dentro del mismo país. Existen colegios o universidades que poseen mejor calidad. Eso guarda relación con las dotaciones iniciales de los individuos $\mathrm{y}$ de los mismos centros educativos. nes. Igualmente, resulta irrealista suponer que los factores institucionales de un país como Colombia sean iguales a los de un país como Singapur ${ }^{18}$. De hecho, existe una gama de estudios que intentan mostrar el efecto que genera sobre el valor productivo del capital humano los factores anteriormente mencionados.

Podemos concluir que existen fallas en la interpretación y medición del capital humano, lo que obliga a abordar la diversidad de estudios que intentan involucrar las asimetrías educativas e institucionales. La primera asimetría busca involucrar en la medición del capital humano, la calidad de la educación. Y la segunda asimetría está relacionada con factores como la burocracia, la corrupción, la concentración del poder en el gobierno, el surgimiento de economías sopesadas en el narcotráfico, el agotamiento de los recursos naturales y el deterioro del medio ambiente, las trabas para la iniciativa privada, la inestabilidad política y la falta de un sistema judicial confiable, las cuales lesionan el valor productivo del capital humano. En las siguientes secciones se amplía estas dos asimetrías.

\section{La calidad de la educación y el capital humano}

Si partimos de los resultados econométricos que la mayoría de los economistas han encontrado, podemos observar que los años promedio de escolaridad de los trabajadores es la variable que estadísticamente mejor se comporta cuando se intenta calcular la contribución del capital humano sobre la producción; no obstante, hay que tener en cuenta que existen problemas significativos asociados con la calidad de la educación recibida, que no son capturados por dicha variable. Veamos algunas razones:

18 Si bien es cierto, que los rezagos de calidad de la educación se pueden mitigar accediendo a una universidad o institución educativa con igual estándar de calidad, bajo el supuesto de que todos los individuos parten bajo las mismas condiciones, es decir, no existen diferencias cognitivas y tampoco en sus dotaciones iniciales, también es cierto que el individuo una vez finalice sus estudios regresará a su país con un capital humano potencial, aunque muy seguramente se enfrentará a una diversidad de problemas de carácter institucional que limitará el valor productivo del capital humano adquirido. 
Por ejemplo, la suposición de que los años promedio de escolaridad es el mejor indicador para medir el capital humano parece dar por supuesto que la educación es igual en todos los países; si esto es así, entonces podríamos concluir que un año de educación en Japón tendría el mismo impacto que un año de escolarización en Tanzania y un título de Harvard o Princeton tendría el mismo valor productivo que un título equivalente de la Universidad de Alaska. Al respecto, Honigher \& Kim (1998) establecen grandes diferencias de calidad en la educación entre los países y muestran que la calidad de la educación está fuertemente relacionada con las diferencias de crecimiento.

El mismo concepto lo expone Steedman (1995), en su estudio que muestra que los niveles de logro social/educativo no son comparables incluso a través de países de la OCDE, debido a las diferencias en la medición y clasificación. En esta misma línea, Hanushenk \& Kim, (1995), muestran que el uso de las tasas de matriculación como una aproximación se basa en la suposición de que los patrones de matrícula no cambian mucho con el tiempo y entre países. Estos autores muestran que existe un problema importante asociado a la calidad de los datos sobre el capital humano. Concluyen que las medidas del nivel formal de la educación son insuficientes y con frecuencia son difíciles de comparar entre países.

Mankiw (2009) expone, que una medida global de capital humano debe tener en cuenta la calidad de la educación y la acumulación de capital humano debido al aprendizaje. El autor abre el debate de la siguiente manera "... un problema que algunos países tienen es la calidad de sus sistemas de enseñanza" (p. 2). El mismo autor también argumenta que los Estados Unidos y otros países desarrollados tienen los mejores sistemas de educación superior, lo que parece explicar por qué los países pobres envían a sus mejores estudiantes al extranjero para obtener grados más altos.

López, Tomás \& Wang (1998), utilizando una muestra de 12 países, encontraron que una fuerza laboral más educada se asocia positivamente con el crecimiento acelerado en las condiciones de la reforma del mercado y un sistema económico orientado hacia el comercio exterior. Y la educación que no contribuye significativamente al crecimiento económico en ausencia de la reforma del mercado de exportación y un sistema productivo orientado, no pueden considerarse productivamente útil. Esto sugiere que el valor productivo del capital humano puede ser calificado, no sólo por la calidad educativa.

La conclusión, es que si los modelos educativos y las normas varían entre los países, no puede decirse que los años promedio de escolaridad de la población en edad de trabajar se traducen en mayores niveles de productividad en proporciones iguales en todos los países y mucho menos en una mejor calidad de vida. Y las diferencias en el valor productivo de la educación no son sino uno de los muchos factores que, insistimos, inciden significativamente en el capital humano.

\section{1. ¿Qué dicen los datos respecto a la escolaridad y calidad de la educación?}

La Tabla 2 muestra el promedio de años de educación de la fuerza laboral y los puntajes obtenidos para 42 países en las pruebas PISA ${ }^{19}$. También incluye el rezago cuantitativo y cualitativo que existe entre el valor máximo de escolaridad (Estados Unidos) y el puntaje máximo de calidad obtenido por Finlandia (544) entre los países que aparecen señalados en la tabla.

19 Se conoce como las pruebas internacionales de educación del Programa de Evaluación Internacional de Estudiantes. PISA no se concentra en una sola materia escolar, sino que revisa las tres áreas de: competencia de lectura, matemáticas y ciencias naturales. 
Tabla 2. Asimetrías educativas y de calidad en 42 países

\begin{tabular}{|c|c|c|c|c|}
\hline País & Escolaridad & *Diferencial Escolaridad & Puntaje de Calidad & *Diferencial Calidad \\
\hline Alemania & 10,2 & $15 \%$ & 510 & $6 \%$ \\
\hline Argentina & 8,8 & $27 \%$ & 396 & $27 \%$ \\
\hline Australia & 10,9 & $9 \%$ & 519 & $5 \%$ \\
\hline Austria & 8,4 & $30 \%$ & 487 & $10 \%$ \\
\hline Bélgica & 9,3 & $23 \%$ & 509 & $6 \%$ \\
\hline Brasil & 4,9 & $59 \%$ & 401 & $26 \%$ \\
\hline Canadá & 11,6 & $3 \%$ & 527 & $3 \%$ \\
\hline Chile & 7,5 & $38 \%$ & 439 & $19 \%$ \\
\hline Colombia & 5,3 & $56 \%$ & 399 & $27 \%$ \\
\hline Dinamarca & 9,7 & $19 \%$ & 499 & $8 \%$ \\
\hline Eslovenia & 7,1 & $41 \%$ & 499 & $8 \%$ \\
\hline España & 7,3 & $39 \%$ & 484 & $11 \%$ \\
\hline Estados Unidos & 12 & $0 \%$ & 496 & $9 \%$ \\
\hline Finlandia & 10 & $17 \%$ & 544 & $0 \%$ \\
\hline Francia & 7,9 & $34 \%$ & 497 & $9 \%$ \\
\hline Grecia & 8,7 & $28 \%$ & 473 & $13 \%$ \\
\hline Holanda & 9,4 & $22 \%$ & 519 & $5 \%$ \\
\hline Hungría & 9,1 & $24 \%$ & 496 & $9 \%$ \\
\hline Indonesia & 5 & $58 \%$ & 385 & $29 \%$ \\
\hline Inglaterra & 9,4 & $22 \%$ & 500 & $8 \%$ \\
\hline Irlanda & 9,4 & $22 \%$ & 497 & $9 \%$ \\
\hline Islandia & 8,8 & $27 \%$ & 501 & $8 \%$ \\
\hline Israel & 9,6 & $20 \%$ & 459 & $16 \%$ \\
\hline Italia & 7,2 & $40 \%$ & 486 & $11 \%$ \\
\hline Japón & 9,5 & $21 \%$ & 529 & $3 \%$ \\
\hline Jordán & 6,9 & $43 \%$ & 402 & $26 \%$ \\
\hline México & 7,2 & $40 \%$ & 420 & $23 \%$ \\
\hline Noruega & 11,8 & $2 \%$ & 500 & $8 \%$ \\
\hline Nueva Zelanda & 11,7 & $3 \%$ & 524 & $4 \%$ \\
\hline Panamá & 8,6 & $28 \%$ & 369 & $32 \%$ \\
\hline Perú & 7,6 & $37 \%$ & 368 & $32 \%$ \\
\hline Polonia & 9,8 & $18 \%$ & 501 & $8 \%$ \\
\hline Portugal & 5,9 & $51 \%$ & 490 & $10 \%$ \\
\hline República Eslovaca & 9,3 & $23 \%$ & 488 & $10 \%$ \\
\hline Singapur & 7 & $42 \%$ & 543 & $0,10 \%$ \\
\hline Suecia & 11,4 & $5 \%$ & 495 & $9 \%$ \\
\hline Suiza & 10,5 & $13 \%$ & 517 & $5 \%$ \\
\hline Tailandia & 6,5 & $46 \%$ & 422 & $22 \%$ \\
\hline Trinidad y Tobago & 7,8 & $35 \%$ & 413 & $24 \%$ \\
\hline Túnez & 5 & $58 \%$ & 392 & $28 \%$ \\
\hline Turquía & 5,3 & $56 \%$ & 454 & $16 \%$ \\
\hline Uruguay & 7,6 & $37 \%$ & 427 & $22 \%$ \\
\hline Promedio & 8,49761905 & 0,291865079 & 470,8413 & 0,133890193 \\
\hline D. Estándar & 1,97366665 & 0,164472221 & 50,82833 & 0,093488818 \\
\hline Correlación & 0,62505613 & & & \\
\hline
\end{tabular}

*Años promedio de Escolaridad 1995. ** Valor obtenido a partir de la diferencia entre el valor máximo y el valor de referencia. Se normalizó por el valor máximo. En los años de Escolaridad el país de referencia es Estados Unidos. Y en la calidad es Singapur.

Fuente: Barro \& Lee (1997). 
En lo que se refiere a la variable años promedio de escolaridad, existen tres rangos. El primer rango oscila entre 12 y 10 años de escolaridad. El segundo rango oscila valores entre 9.8 y 7 . Y el último rango entre 6.9 y 4.9. La primera cohorte la lidera los Estados Unidos (12), seguido por Nueva Zelanda (11.8), Noruega (11.7), Canadá (11.6) y Suecia (11.4), Australia (10.9) y Alemania (10.2) y Finlandia (10.0). En la segunda cohorte aparecen con los mayores valores: Polonia (9.8), Dinamarca (9.7), Israel (9.6), Japón (9.5), Irlanda (9.4), Inglaterra (9.4), Holanda (9.4), Republica Eslovaquia (9.3), y Hungría (9.1). Los países con los más bajos niveles de escolaridad son: Brasil (4,9), Túnez (5.0), Indonesia (5.0), Turquía (5.3), Colombia (5.3) y Portugal (5.9)

En cuanto al rezago de calidad, con excepción de Túnez (28\%), Trinidad Tobago, Tailandia e Indonesia, los países latinoamericanos arrojaron la mayor diferencia: Panamá (32\%), Perú (32\%), Argentina $(27 \%)$, Colombia (27\%), Brasil (26\%), México (23\%), y Uruguay (22\%), mientras que los menores rezagos fueron obtenidos por Singapur $(0.1 \%)$ y Japón (3\%). Los países que consiguieron los más altos niveles de escolaridad, arrojaron una diferencia en la calidad educativa que osciló entre el 3 y 9\%.

\subsection{Factores institucionales}

Además del problema de la calidad de la educación, existen otros elementos que afectan el valor productivo del capital humano. Nos referimos específicamente a factores como inestabilidad política, social e institucional, corrupción, narcotráfico, pobreza, entre otros, los cuales juegan un rol importante para determinar el producto final de la actividad humana $y$, por lo tanto, deben tenerse en cuenta en cualquier intento serio de medir el impacto del capital humano sobre el PIB. A estos factores los denominaremos factores de resistencia institucional.

Al respecto, existe una serie de trabajos empíricos que muestran cómo la corrupción, las regulaciones gubernamentales, trámites burocráticos, e incluso la fortaleza de los sistemas legislativos y judiciales, reducen la actividad humana en el sistema económico y social de una sociedad. Un estudio realizado por Mauro (1997), mostró que la ineficiencia insti- tucional (inestabilidad institucional, política y social, la falta de eficiencia y la integridad en el sistema judicial, la burocracia, el ambiente institucional para hacer negocios y la corrupción) vedan tanto la inversión en recursos humanos y físicos como el crecimiento económico ${ }^{20}$. En palabras de Mauro (1997): "La asociación negativa entre la corrupción y la inversión, así como el crecimiento, es importante tanto desde el punto de vista estadístico como económico. Hay una correlación positiva y significativa entre los índices de eficiencia burocrática y la estabilidad política..." (p. 705). En definitiva, lo que Mauro (1997)encuentra es un efecto combinado entre el capital humano y el crecimiento económico que resulta del entorno cultural en el que se desarrolla la actividad humana.

En el mismo camino apunta Blanchard (1997), cuando esgrime que problemas como la corrupción, la violencia, los conflictos armados, el tráfico de drogas, los derechos de propiedad mal definidos, la inestabilidad política, la falta de emprendedores de negocios, mercados financieros poco desarrollados y otros, son obstáculos significativos a la eficacia de capital humano ${ }^{21}$. Por su parte, PakHung Mo (2001) encuentra en su trabajo sobre corrupción -uno de los factores de resistencia considerados aquí- y crecimiento, que un incremento del $1 \%$ en los niveles de corrupción reduce la tasa de crecimiento del PIB en $0.72 \%$. Y el más importante canal a través del cual la corrupción afecta el crecimiento, es la inestabilidad política (53\% del efecto total). Rogers (2008), en un estudio de corte transversal, calcula un índice de corrupción de países, con submuestras de alta y baja corrupción, y sugiere que el impacto del capital humano es mayor en la submuestra de los países de baja corrupción en comparación con la submuestra de países de alta corrupción.

20 Aunque Mauro (1995) identificó cinco factores, sospechamos que puede haber muchos otros; sin embargo, los cinco identificados serán considerados en nuestra investigación para calcular el índice global de capital humano.

21 Una verdadera multitud de estos factores limitantes abunda en los países y principalmente en los del Tercer Mundo $y$ se encuentran entre las principales preocupaciones que nos referimos en este trabajo como factores de resistencia institucional 
Los anteriores hallazgos sugieren que el capital humano debe considerarse desde una perspectiva más amplia de la que normalmente trabajan los economistas. Así, factores como por ejemplo, las políticas económicas y la planificación industrial y social que tienen lugar dentro de la sociedad nacional, como otras circunstancias, definen la calidad del trabajo y determinan el rendimiento de los empleados dentro del lugar de trabajo ${ }^{22}$. La presencia de estas condiciones, que en conjunto definen el contexto organizacional y comunitario de trabajo y la producción, propone que la forma como la sociedad humana está organizada tiene mucho que ver con las diferencias en el crecimiento económico de los países ${ }^{23}$.

De hecho, si observamos los países tercermundistas, nos damos cuenta que el desarrollo económico de estos tiende a ser producto de una planificación errática e impredecible, cimentada en "obstáculos" para la eficacia del capital humano, lo que implica alteraciones en la interacción entre el nivel y la calidad de la enseñanza, comúnmente asociada con el termino capital humano, y el producto final que es la producción ${ }^{24}$. De modo, que una sociedad

22 Algunos de ellos tienen que ver con regulaciones tales como las leyes de salario mínimo, las normas y requisitos que se ocupan de calificaciones profesionales y técnicas, políticas educativas, la planificación industrial, políticas de beneficios de desempleo, los estatutos de seguridad social y demás. Otros tienen que ver con la eficiencia o la insuficiencia del aparato administrativo del Estado, la estabilidad política, el entorno empresarial, es decir, las políticas estatales con respecto a registrarse negocio y el papeleo necesario, las leyes fiscales y demás.

23 Esto puede ser considerado, no sólo desde el punto de vista de las influencias socioculturales que afectan el desempeño del trabajador, sino también desde el punto de vista de las políticas económicas y la planificación industrial y social que tienen lugar dentro de la sociedad nacional, así como otras circunstancias que juntos definen la calidad del trabajo y determinan el rendimiento de los empleados dentro del lugar de trabajo.

24 Estas situaciones conducen a un deterioro de la confianza pública en el sistema político y administrativo y de la aceptación general de una tradición que permite hacer sobornos y otras medidas corruptas para lograr sus fines. Esta realidad erosiona aún más la confianza pública en el estado, sin embargo, y se traduce en disminuciones agudas de capital social y el debilitamiento del esfuerzo económico colectivo. El escritor colombiano Juan Sudanski afirma que que presenta este tipo de problemas en su entorno social, político y económico, constituyen un obstáculo colosal para el bienestar social y el progreso económico.

\subsection{Estadísticas sobre asimetrías institucionales}

En la tabla No 3, se presentan las asimetrías institucionales para 37 países. El país de referencia o comparación es Singapur, que según el estudio de Mauro (1997) recibió una calificación de 10 en los factores Estabilidad política, sistema judicial, corrupción, restricciones para iniciativas empresariales, y burocracia. En un análisis general, las mayores asimetrías en los cincos factores las presentan Indonesia, Tailandia, Corea, Brasil, Argentina, España, Grecia, Italia, Colombia, Perú, Panamá, Uruguay, Turquía y Portugal.

sin el capital social, el capital humano no existe. "En los sistemas de este tipo, el esfuerzo humano no es recompensado por cualquier excelencia intrínseca sino por su desviación y perversión y esto hará que el capital humano luzca totalmente ineficaz.". 
Tabla 3. Asimetrías institucionales en 37 países

\begin{tabular}{|c|c|c|c|c|c|c|c|c|c|c|}
\hline País & $\begin{array}{l}\text { E. Polí- } \\
\text { tica }\end{array}$ & $\begin{array}{l}\text { Diferen- } \\
\text { cial }\end{array}$ & $\begin{array}{l}\text { S. Judi- } \\
\text { cial }\end{array}$ & $\begin{array}{l}\text { Diferen- } \\
\text { cial }\end{array}$ & $\begin{array}{l}\text { Corrup- } \\
\text { ción }\end{array}$ & $\begin{array}{l}\text { Diferen- } \\
\text { cial }\end{array}$ & $\begin{array}{l}\text { Red } \\
\text { Tape }\end{array}$ & $\begin{array}{l}\text { Diferen- } \\
\text { cial }\end{array}$ & $\begin{array}{c}\text { Burocra- } \\
\text { cia }\end{array}$ & $\begin{array}{l}\text { Diferen- } \\
\text { cial }\end{array}$ \\
\hline Alemania & 9 & 0,1 & 7,5 & 0,25 & 9,5 & 0,05 & 8,21 & 0,18 & \begin{tabular}{|l|l|}
8,67 \\
\end{tabular} & 0,13 \\
\hline Argentina & 6 & 0,4 & 6,66 & 0,33 & 7,66 & 0,23 & 7,72 & 0,23 & 6,77 & 0,32 \\
\hline Australia & 10 & 0 & 9,25 & 0,08 & 10 & 0 & 8,5 & 0,15 & 9,75 & 0,03 \\
\hline Austria & 9,5 & 0,05 & 7,25 & 0,28 & 8 & 0,2 & 9,04 & 0,1 & \begin{tabular}{|l|}
8,25 \\
\end{tabular} & 0,18 \\
\hline Bélgica & 9,5 & 0,05 & 8 & 0,2 & 9,75 & 0,03 & 8 & 0,2 & 9,08 & 0,09 \\
\hline Brasil & 5,75 & 0,43 & 4 & 0,6 & 5,75 & 0,43 & 7,54 & 0,25 & 5,17 & 0,48 \\
\hline Canadá & 9,25 & 0,08 & 9,5 & 0,05 & 10 & 0 & 9 & 0,1 & 9,58 & 0,04 \\
\hline Chile & 7,25 & 0,28 & 9,25 & 0,08 & 9,25 & 0,08 & 6,46 & 0,35 & 8,58 & 0,14 \\
\hline Colombia & 7,25 & 0,28 & 4,5 & 0,55 & 4,5 & 0,55 & 6 & 0,4 & 5,42 & 0,46 \\
\hline Corea & 7,5 & 0,25 & 6 & 0,4 & 5,8 & 0,42 & 6,5 & 0,35 & 6,1 & 0,39 \\
\hline Dinamarca & 10 & 0 & 9,5 & 0,05 & 9,25 & 0,08 & 8,5 & 0,15 & 9,58 & 0,04 \\
\hline España & 6,25 & 0,38 & 6 & 0,4 & 7 & 0,3 & 6,67 & 0,33 & 6,42 & 0,36 \\
\hline $\begin{array}{l}\text { Estados } \\
\text { Unidos }\end{array}$ & 10 & 0 & 9,25 & 0,08 & 10 & 0 & 9,33 & 0,07 & 9,75 & 0,03 \\
\hline Finlandia & 10 & 0 & 8,5 & 0,15 & 9,5 & 0,05 & 8,79 & 0,12 & 9,33 & 0,07 \\
\hline Francia & 8 & 0,2 & 6,75 & 0,33 & 10 & 0 & 8,92 & 0,11 & 8,25 & 0,18 \\
\hline Grecia & 7 & 0,3 & 4 & 0,6 & 6,25 & 0,38 & 8,63 & 0,14 & 5,75 & 0,43 \\
\hline Holanda & 10 & 0 & 10 & 0 & 10 & 0 & 8,83 & 0,12 & 10 & 0 \\
\hline Indonesia & 2,5 & 0,75 & 2,75 & 0,73 & 1,5 & 0,85 & 7,46 & 0,25 & 2,25 & 0,78 \\
\hline Inglaterra & 10 & 0 & 7,75 & 0,23 & 9,25 & 0,08 & 8,33 & 0,17 & 9 & 0,1 \\
\hline Irlanda & 8,75 & 0,13 & 7,5 & 0,25 & 9,75 & 0,03 & 7,67 & 0,23 & 8,67 & 0,13 \\
\hline Israel & 10 & 0 & 7,5 & 0,25 & 9,25 & 0,08 & 6,25 & 0,38 & 8,92 & 0,11 \\
\hline Italia & 6,75 & 0,33 & 4,75 & 0,53 & 7,5 & 0,25 & 7,92 & 0,21 & 6,33 & 0,37 \\
\hline Japón & 10 & 0 & 8,5 & 0,15 & 8,75 & 0,13 & 9,42 & 0,06 & 9,08 & 0,09 \\
\hline Jordania & 8,66 & 0,13 & 6,33 & 0,37 & 8,33 & 0,17 & 7,78 & 0,22 & 7,77 & 0,22 \\
\hline México & 6 & 0,4 & 5,25 & 0,48 & 3,25 & 0,68 & 6,88 & 0,31 & 4,83 & 0,52 \\
\hline Noruega & 10 & 0 & 9 & 0,1 & 10 & 0 & 9,5 & 0,05 & 9,67 & 0,03 \\
\hline Nueva Zelanda & 10 & 0 & 10 & 0 & 10 & 0 & 8,5 & 0,15 & 10 & 0 \\
\hline Panamá & 6,75 & 0,33 & 7,25 & 0,28 & 5 & 0,5 & 7,54 & 0,25 & 6,33 & 0,37 \\
\hline Perú & 6,75 & 0,33 & 5,75 & 0,43 & 7,25 & 0,28 & 6,04 & 0,4 & 6,58 & 0,34 \\
\hline Portugal & 5,5 & 0,45 & 4,5 & 0,55 & 6,75 & 0,33 & 7,54 & 0,25 & 5,58 & 0,44 \\
\hline Singapur & 10 & 0 & 10 & 0 & 10 & 0 & 10 & 0 & 10 & 0 \\
\hline Suecia & 10 & 0 & 8,5 & 0,15 & 9,25 & 0,08 & 9 & 0,1 & 9,25 & 0,08 \\
\hline Suiza & 10 & 0 & 10 & 0 & 10 & 0 & 9,25 & 0,08 & 10 & 0 \\
\hline Tailandia & 3,25 & 0,68 & 3,25 & 0,68 & 1,5 & 0,85 & 5,63 & 0,44 & 2,67 & 0,73 \\
\hline $\begin{array}{l}\text { Trinidad / } \\
\text { Tobago } \\
\end{array}$ & 8 & 0,2 & 4 & 0,6 & 6,5 & 0,35 & 7,79 & 0,22 & 6,17 & 0,38 \\
\hline Turquía & 4 & 0,6 & 5,33 & 0,47 & 6 & 0,4 & 8,17 & 0,18 & 5,11 & 0,49 \\
\hline Uruguay & 6,5 & 0,35 & 6 & 0,4 & 8 & 0,2 & 9 & 0,1 & 6,83 & 0,32 \\
\hline $\operatorname{Max}$ & 10 & 0 & 10 & 0 & 10 & 0 & 10 & 0 & 10 & 0 \\
\hline Min & 2,5 & & 2,75 & & 1,5 & & 5,63 & & 2,25 & \\
\hline
\end{tabular}

Fuente: Paolo Mauro (1997). Cálculos Propios 
Como era de esperarse, las menores asimetrías las presentaron los países que ostentan los mayores índices de escolaridad o de años promedio de educación de los trabajadores. Esto supondría que existe una relación estrecha entre las asimetrías institucionales y las educativas.

Desagregando por factores, se encuentra que Indonesia, Tailandia, México y Colombia presentan la mayor asimetría por corrupción. Los países más inestables políticamente son: Tailandia, Indonesia y Turquía. Los peores sistemas judiciales los tienen Brasil Grecia, Trinidad Tobago y Tailandia. Los países que ponen los mayores obstáculos a las iniciativas empresariales son Tailandia, Perú y Colombia. Y, los países más burocráticos son Indonesia, Tailandia, Corea, Portugal, Grecia, Colombia, México, Brasil.

\section{Construcción de un indicador Global de Capital Humano (IGCH)}

Los problemas expuesto en este trabajo muestran que es necesario construir un Indicador Global de Capital Humano (IGCH), que involucre los años promedio de escolaridad de los trabajadores, y se agreguen los rezagos de calidad en la educación y los llamados factores de resistencia institucional, que son los que condicionan el valor productivo del capital humano.

La lógica general que planteamos aquí es que un individuo desarrolla nuevas ideas partiendo de unas dotaciones iniciales -por ejemplo, nivel educativo de los padres- y unas habilidades cognitivas que son fortalecidas con el tipo y calidad de educación que adquiere y con las condiciones institucionales o el medio que lo circundan. De modo que, el cálculo de un IGCH deberá incluir, además de los años promedio de educación de los trabajadores, variables que tienen que ver con las asimetrías en la calidad de la educación y factores institucionales. Partiendo de esta lógica, a continuación se calcula un IGCH incorporando las variables años de escolaridad, calidad de la educación, estabilidad política, sistema judicial, corrupción, dificultad para poner en marcha nuevas ideas y burocracia.

\subsection{Metodología para calcular el indicador}

La metodología propuesta para calcular el IGCH, se elabora a partir de una técnica multivariada de análisis factorial. Esta técnica se emplea debido a que es la manera más eficiente de reducir la dimensionalidad a partir de la información recolectada y sin incluir ningún tipo de sesgo por parte del investigador. De la misma forma, la ponderación de cada una de las variables dentro del indicador es producto del grado de correlación que comparten con las otras variables; en este sentido, la estructura del indicador es el resultado de la comunalidad de las variables que lo componen.

El modelo matemático en su representación matricial es como sigue: suponga que las variables que conforman el indicador es $\mathrm{K}$, de modo que se tiene el vector $\mathrm{P}$ de dimensión $\mathrm{K} \times 1$. Estas variables se transformaran en $\mathrm{K}$ factores tales que cada factor es una combinación lineal de la $\mathrm{K}$ variables originales. En este sentido, existe también una matriz de dimensión $\mathrm{K} \times \mathrm{K}$ que contiene las ponderaciones óptimas de cada variable dentro del k-ésimo factor, en símbolos:

$\mathbf{P}=\left[\begin{array}{c}\mathrm{P}_{1} \\ \mathrm{P}_{2} \\ \mathrm{P}_{3} \\ \vdots \\ \mathrm{P}_{K}\end{array}\right] \quad \mathbf{F}=\left[\begin{array}{c}\mathrm{F}_{1} \\ \mathrm{~F}_{2} \\ \mathrm{~F}_{3} \\ \vdots \\ \mathrm{F}_{K}\end{array}\right] \quad \mathrm{A}=\left[\begin{array}{cccc}\lambda_{11} & \lambda_{12} & \ldots & \lambda_{1 K} \\ \lambda_{21} & \lambda_{22} & \ldots & \lambda_{2 K} \\ \vdots & \vdots & \ldots & \vdots \\ \lambda_{K 1} & \lambda_{K 2} & \ldots & \lambda_{K K}\end{array}\right] \lambda_{k}=\left[\begin{array}{c}\lambda_{k 1} \\ \lambda_{k 2} \\ \lambda_{k 3} \\ \vdots \\ \lambda_{k K}\end{array}\right] ; \square k: 1 \ldots K$

Sea entonces

$\mathrm{F}=\mathrm{AP}$, donde $\mathrm{F}_{1}=\lambda^{T}{ }_{1} \mathrm{P}=\lambda_{11} \mathrm{P}_{1}+\lambda_{12} \mathrm{P}_{2}+\ldots+\lambda_{1 K} \mathrm{P}_{K}$

Dado que las variables que componen cada índice tienen diferentes métricas, entonces se estandarizarán para poder compararlas entre sí y para construir la matriz de correlaciones que se analizará a través del método de componentes principales ${ }^{25}$.

25 El Análisis de Componentes Principales (ACP) es una técnica estadística de síntesis de la información, o reducción de la dimensión (número de variables). Es decir, ante un banco de datos con muchas variables, el objetivo será reducirlas a un menor número perdiendo la menor cantidad de información posible. Los nuevos componentes principales o factores serán una combinación lineal de las variables originales, y además serán independientes entre sí. Un aspecto clave en 
En este sentido sea $E[P]=0$ y la matriz de varianza y covarianzas $\mathrm{V}(\mathrm{P})=E\left[\mathrm{P} \mathrm{P}^{T}\right]=\mathrm{S}=(1 / n) \mathrm{P}^{T} \mathrm{P}$. Por lo tanto $E[\mathrm{AP}]=\mathrm{AE}[\mathrm{P}]=0 \mathrm{y} \mathrm{V}(\mathrm{F})=\mathrm{V}(\mathrm{AP})=\mathrm{E}[\mathrm{AP})$ $\left.\mathrm{AP})^{T}\right]=\mathrm{ASA}^{T}$. De modo que $\operatorname{var}(\mathrm{F} 1)=\left(\mathrm{ASA}^{T}\right)_{11}=$ $\lambda^{T}{ }_{1} S \lambda_{1}$.

El Indicado Global de Capital Humano (t) se estima maximizando la varianza de $\mathrm{F}_{1}$, que será el primer factor obtenido utilizando el método de componentes principales. En símbolos, el índice t resulta de maximizar la siguiente expresión:

$\operatorname{Max} \operatorname{var}\left(\mathrm{F}_{1}\right)=\lambda^{T_{1} S} \lambda_{1}$

Sujeto a $\lambda^{T_{1}} \lambda_{1}=1$

Este problema de optimización se puede resolver a través del método de multiplicadores de Lagrange como sigue a continuación:

$L\left(\lambda_{1}, U\right)=\lambda^{T}{ }_{1} S \lambda_{1}-U\left(\lambda{ }^{T}{ }_{1} \lambda_{1}-1\right)$. Las condiciones de primer orden (CPO) de este problema son:

$\mathbb{\|} L(x) / \Phi \lambda_{1}=2 S \lambda_{1}-2 U \lambda_{1}=0, S \lambda_{1}=U \lambda_{1}$

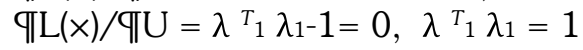

La ecuación resultante de la primera CPO corresponde a una descomposición matricial bien conocida que tiene dos componentes claves a lado y lado de la ecuación, U conocido como un valor propio de la matriz $S$ y $\lambda$ el cual es un vector propio asociado inequívocamente con $U$.

Después de maximizar se puede probar que var $\left(\mathrm{F}_{1}\right)$ $=\lambda^{T_{1}} U \lambda_{1}=\mathrm{U}$; por lo tanto, el problema se resuelve al encontrar el máximo valor propio de la matriz $\mathrm{S}$. Por último, $\mathrm{F}_{1}$ se podrá estimar a través de las cargas factoriales contenidas en el vector propio $\lambda_{1}$.

En resumen, $\mathrm{F}_{1}$ se puede interpretar como una forma de reducir la dimensionalidad a través de la maxi-

ACP es la interpretación de los factores, ya que ésta no viene dada a priori, sino que será deducida tras observar la relación de los factores con las variables iniciales (habrá, pues, que estudiar tanto el signo como la magnitud de las correlaciones). Esto no siempre es fácil, y será de vital importancia el conocimiento que el experto tenga sobre la materia de investigación. mización de la varianza que comparten las variables que componen el índice $t$, lo que significa que $F_{1}$ es una proyección optima de la varianza que resulta cuando se combinan linealmente las variables bajo análisis. Una vez se obtiene $\mathrm{F}_{1}$ este vector se reparametriza para proyectarlo en una escala continua [0,1], el cual será el indicador t. En esta escala, 0 representa el valor mínimo de Capital Humano y 1 su valor máximo.

\subsection{Consideraciones Metodológicas}

El cálculo del IGCH se efectuó mediante el uso del método de componentes principales. Primero se seleccionaron los países que presentaron información de las 7 variables consideradas -años promedio de educación de los trabajadores (ESC), puntaje en la prueba de matemáticas y ciencia (PCM), estabilidad política (EP), sistema judicial (SJ), corrupción (CO), nivel de obstáculo para iniciativas Empresariales (NOIE) y burocracia (BU)-. El total de países que cumplió dicha condición fue de 37.

Dado que el Test de Barlett ${ }^{26}$ rechaza la hipótesis nula de la no pertinencia del modelo Factorial, es factible hacer componentes principales. La medida Kaiser Meyer-Olkin ${ }^{27}$ fue de 0,825. Se escogió el primer componente, ya que explica el $76 \%$ de varianza. Posteriormente, cada coeficiente se normalizó por método de escalonamiento óptimo ${ }^{28}$ y se obtuvo la siguiente ecuación:

$$
\begin{aligned}
\text { Factor } & =0,139 E S C_{i}+0.122 P_{C M}+0,154 E P_{i}+0,153 S J_{i} \\
& +0118 C O_{i}+0,153 N O I E+0,162 \mathrm{BU}_{i}
\end{aligned}
$$

$26 \quad$ El Test de Bartlett es un contraste de hipótesis de igualdad de desviaciones estándar. Muy utilizado en ANOVA, donde se requiere homogeneidad de varianzas.

27 La medida de adecuación muestral de Kaiser-Meyer-Olkin nos indica que la muestra de datos en su conjunto es adecuada dado que este coeficiente oscila entre 0 y 1 , de manera que cuanto más cercano se encuentra de 1 más adecuados son los datos. Por otro lado, la Prueba de esfericidad de Bartlett intenta probar la hipótesis de que la matriz de correlaciones es o no una matriz identidad, lo que implicaría un nivel nulo de correlación entre las variables. En este caso, dado la significación 0.000 , tenemos evidencia empírica suficiente como para rechazar la hipótesis de que dicha matriz sea una matriz identidad.

28 El escalamiento óptimo también revela relaciones no lineales 
Tabla 4. Indicador Global de Capital Humano (IGCH) de 37 países

\begin{tabular}{|l|l|l|}
\hline \multicolumn{1}{|c|}{ PAIS } & \multicolumn{1}{|c|}{ IGCH } & \multicolumn{1}{c|}{ * IGCH1 } \\
\hline Alemania & 0,74471 & 0,81249 \\
\hline Argentina & 0,49744 & 0,51251 \\
\hline Australia & 0,85989 & 0,95223 \\
\hline Austria & 0,70234 & 0,76109 \\
\hline Bélgica & 0,75224 & 0,82162 \\
\hline Brasil & 0,29664 & 0,2689 \\
\hline Canadá & 0,88147 & 0,9784 \\
\hline Chile & 0,58987 & 0,62464 \\
\hline Colombia & 0,27865 & 0,24708 \\
\hline Corea & 0,56494 & 0,5944 \\
\hline Dinamarca & 0,81779 & 0,90115 \\
\hline España & 0,45104 & 0,45622 \\
\hline E. Unidos & 0,89917 & 0,99988 \\
\hline Finlandia & 0,83372 & 0,92048 \\
\hline Francia & 0,67975 & 0,73368 \\
\hline Grecia & 0,49059 & 0,5042 \\
\hline Holanda & 0,86315 & 0,95618 \\
\hline Indonesia & 0,07498 & 0 \\
\hline Inglaterra & 0,75749 & 0,828 \\
\hline & & \\
\hline
\end{tabular}

\begin{tabular}{|l|l|l|}
\hline \multicolumn{1}{|c|}{ PAIS } & \multicolumn{1}{|c|}{ IGCH } & \multicolumn{1}{c|}{ *IGCH1 } \\
\hline Irlanda & 0,7018 & 0,76043 \\
\hline Israel & 0,66046 & 0,71028 \\
\hline Italia & 0,48294 & 0,49492 \\
\hline Japón & 0,8232 & 0,9077 \\
\hline Jordán & 0,54373 & 0,56867 \\
\hline México & 0,31805 & 0,29488 \\
\hline Noruega & 0,89632 & 0,99642 \\
\hline N. Zelanda & 0,89927 & 1 \\
\hline Panamá & 0,45567 & 0,46183 \\
\hline Perú & 0,38761 & 0,37927 \\
\hline Portugal & 0,38904 & 0,381 \\
\hline Singapur & 0,86981 & 0,96425 \\
\hline Suecia & 0,83877 & 0,9266 \\
\hline Suiza & 0,89872 & 0,99933 \\
\hline Tailandia & 0,09335 & 0,02228 \\
\hline T y Tobago & 0,44597 & 0,45007 \\
\hline Turquía & 0,34786 & 0,33105 \\
\hline Uruguay & 0,53699 & 0,56049 \\
\hline & & \\
\hline
\end{tabular}

* Normalizado con el método de escalamiento óptimo.

Años promedio de Escolaridad 1995. Fuente Barro \& Lee (1997) y Mauro (1999).

Fuente: Cálculos Propios.

En la tabla No 4 se presenta el indicador Global de Capital Humano estimado para los 37 países. En general, los resultados muestran a los países industrializados con IGCH que se aproxima a la uni$\mathrm{dad}^{29}$. Entre los más altos se destacan: Nueva Zelanda (0,89927), Estados Unidos (0.89917), Suiza $(0,89872)$, Canadá $(0,88147)$, Holanda $(0,86315)$, Noruega $(0,89632)$, Singapur $(0,86981)$ (Australia $(0,85989)$, Suecia $(0,83877)$, Finlandia $(0,83372)$. Japón $(0,82320)$ y Dinamarca $(0,81799)$. Estos resultados son congruentes debido a que estos países

29 En este caso se está analizando el IGCH sin normalización. El normalizado es el que tiene una escala de 0 a 1. presentan los mayores niveles promedio de escolaridad, los mayores puntajes en las pruebas PISA y sobre todo, poseen un nivel muy bajo de corrupción, burocracia y restricciones mínimas para iniciativas empresariales. También gozan de sistemas políticos estables y sistemas judiciales confiables.

No ocurre igual con la mayoría de los países latinoamericanos, Indonesia y Tailandia, que presentan un indicador Global de Capital Humano que tiende a cero. En cuanto a los países latinoamericanos, Brasil $(0,296640)$ y Colombia $(0,27865)$ muestran los valores más bajos. Chile arrojó el mayor valor $(0,58987)$, indicando que es un país que tiende a superar sus problemas de educación y sus restric- 
ciones institucionales que se requiere para que el capital humano pueda aportar un valor productivo cercano a los obtenidos por Francia e Inglaterra ${ }^{30}$.

\section{Conclusiones}

A pesar de la importancia que representa para el crecimiento del PIB el acervo de capital humano, no existe consenso entre los economistas, sobre cuál es la variable -proxy- que mejor explica este factor de producción. Aunque, los años promedio de educación de los trabajadores es la variable más frecuentemente utilizada para capturar la contribución del capital humano sobre el producto, los cálculos econométricos, expresados en elasticidades, arrojan diferencias significativas entre los diferentes estudios más conocidos sobre el tema.

Los países que tienen los más altos niveles de escolaridad, no necesariamente obtienen los mayores puntajes en las pruebas de matemáticas y ciencias; en otras palabras, un mayor nivel de escolaridad no se traduce en una mayor calidad. Este resultado se obtiene al menos con las pruebas PISA; habría que mirar los resultados cuando se toma como estándar de medición la calidad de las universidades.

Una vez tenidos en cuenta los problemas de medición del capital humano y los factores de resistencia institucional y calculado el IGCH, nuestros resultados muestran que los países con mayor nivel de desarrollo poseen un IGCH que se aproxima a la unidad, lo que deja entrever que existe una alta simbiosis entre los años promedio de educación de los trabajadores, la calidad de la educación y los factores institucionales; es decir, que las asimetrías educativas e institucionales tienden a ser mínimas entre los países de mayor desarrollo. En efecto, entre los países con mayores IGCH destacan Nueva Zelanda (0,89927), Estados Unidos (0.89917), Suiza (0,89872), Canadá $(0,88147)$, Holanda $(0,86315)$, Noruega $(0,89632)$, Singapur $(0,86981)$, Australia $(0,85989)$, Suecia $(0,83877)$, Finlandia $(0,83372)$,

30 Hay que tener cuidado con analizarlo de esa manera, actualmente existen países en el ranking en comparación con hace diez años. Si sólo se mira el ranking, parece que Chile va hacia atrás, pero en realidad se van añadiendo más países.
Japón $(0,82320)$ y Dinamarca $(0,81799)$. En cuanto a nivel de Latinoamérica, Brasil $(0,296640)$ y Colombia $(0,27865)$ presentan los valores más bajos del indicador IGCH. Entre este último grupo de países, Chile presentó el mayor valor $(0,58987)$, indicando que éste país viene fortaleciendo su sistema educativo $e$ institucional.

Los resultados de esta investigación aportan evidencia acerca de la idea de que las diferencias asimétricas, tanto educativas como institucionales, entre los países están asociadas con los altos niveles de corrupción, burocracia y, en general, problemas institucionales; al igual que con la presencia de sistemas educativos rígidos y anticuados, que no permiten la generación de nuevas ideas y aporte significativo a la producción ${ }^{31}$.

En un mundo globalizado y altamente competitivo, los resultados de esta investigación son preocupantes, porque si no se corrigen los problemas detectados la brecha entre los países desarrollados y atrasados continuará ampliándose. En este contexto, una recomendación que se deriva de esta investigación es que, la prioridad de los países que arrojaron altas asimetrías educativas e institucionales es avanzar hacia un sistema educativo competitivo y bajar los niveles de corrupción, burocracia, y restricción para iniciativas empresariales.

Finalmente, teniendo en cuenta que: primero, no existe un consenso sobre qué es realmente el capital humano y cómo medirlo; segundo, el valor productivo del capital humano depende de la organización del sistema económico, de las habilidades individuales, y de muchas otras consideraciones; y, tercero, los años promedios de escolaridad no es una medida apropiada para hacer comparaciones entre países -debido a que los costos de producción de la educación y la productividad varían de un país a otro (Becker, 1998)-, se sugiere hacer, en un trabajo posterior, un contraste entre el IGCH y los años promedio de educación de los trabajadores (APE) para una muestra amplia de países, con el objeto de

31 Para una mayor extensión del tema véase Sáenz \& Díaz (2010). 
determinar ¿qué medida ofrece mejor ajuste estadístico?, en aras de ofrecer una nueva definición del cuestionado capital humano, involucrando las asimetrías educativas e institucionales que caracterizan o hacen diferente a cada país.

\section{Referencias}

Acemoglu, D. \& Verdier, T. (1998). Property Rights, Corruption and the Allocation of Talent: A General Equilibrium Approach. Econ. J. 108 (450):1381-1403.

Barro, J. R. (1991). Economic Growth in a Cross Section of Countries. Quarterly Journal of Economics, CVI (May): 407-43

Blanchard, O. (1997). Microeconomic, Ed. Prentice Hall.

Caballé, J. \& Santos, M.S., (1993). On endogenous growth with physical and human capital. Journal of Political Economy 101: 1042-1067.

Caselli, F., Esquivel, G. \& Lefort, F. (1996). Reopening the Convergence Debate: A New Look at Cross-Country Growth Empirics. Journal of Economics Growth, 1: 363-389.

Dessus S. (2001). Human capital and growth the recovered role of educational systems. World Bank

Dornbusch, R., Fischer S. \& R. Startz (1998), Macroeconomía, McGraw-Hill, Séptima Edición, Cap. 1.

Johannes, H. (1998). Human capital and economic growth a survey of the literatura. CPB Report

Barro R. \& Lee, J. (1997). "Schooling Quality in a Cross-Section of Countries," forthcoming, NBER Working Paper

Lucas, R. E. Jr., (1988). On the Mechanics of Development Planning. Journal of Monetary Economics 22: 3-42.
Mankiw G. Macroeconomía. (2009) 5 a . Edición. Cengage Learning editores. México D.F.

Mankiw. N., Romer, D. \& Weil, D. (1992). A Contribution to the Empirics of Economic Growth. The Quarterly Journal of Economics, 107 (2): 407-437

Mauro, P. (1995). Corruption and Growth. Quarterly Journal Economics 110 (3)

Mauro, P. (1997). The effects of corruption on growth, investment, and government expenditure: A cross-country analysis. Institute International for Economics.

Pritchett, L. (1996). Where Has All the Education Gone?. Policy, Research working paper; no. WPS 1581. Washington, DC: World Bank.

Rebelo, S. (1991). Long-Run Policy Analysis and the Long-Run Growth. Journal of Political Economy 99 (3): 500-521.

Reveiz, E. (2004). El desenlace neoliberal: tragedia o renacimiento.

Rogers, M. (2008). Directly Unproductive Schooling: How Country Characteristics Affect the Impact of Schooling on Growth. European Economic Review, 52: 356-385.

Romer, P. (1986). Increasing Returns and Long-Run Growth. Journal of Political Economy,

Romer, P. (1990). Endogenous Technological Change. Journal of Political Economy 98(14): 71-102.

Sáenz, J. \& Díaz, A. (2010). Evaluation of Educational System, Including Peer Instruction, Educational Levels and Degree s of Freedom, Working paper

Shultz T.W. (1961). Investment in Human Capital. America Economic Review. 51: 14-15

Uzawa, H. (1965). Optimal technical change in an aggregative model of economic growth. International Economic Review 6: $18-31$ 